\title{
CHARACTERISTIC CYCLES AND DECOMPOSITION NUMBERS
}

\author{
Kari Vilonen and Geordie Williamson
}

\begin{abstract}
We observe that the characteristic cycle of a D-module gives bounds for decomposition numbers of intersection cohomology complexes.
\end{abstract}

\section{Introduction}

In the papers [9] and [11] Kazhdan and Lusztig made conjectures that amount to the irreducibility of characteristic varieties of intersection cohomology sheaves of Schubert varieties in type $A_{n}$. In [6] Kashiwara and Saito provide a counterexample to this conjecture for $n=7$. Previously Kasiwara and Tanisaki $[8,15]$ gave examples of reducible characteristic varieties in other types. On the other hand, in the context modular representation theory, Braden discovered examples of non-trivial decomposition numbers for perverse sheaves (with coefficients in a finite field) on flag varieties. One of these examples occurs in type $A_{7}$. The second author [16] pointed out recently that similar considerations give a counterexample to the Kleshchev-Ram conjecture [10].

The purpose of this paper is to observe that this is no accident. Given a sheaf with integer coefficients extension of scalars yields a sheaf with rational coefficients and modular reduction yields a sheaf with coefficients in any finite field. It is an easy observation that all the sheaves in question have the same characteristic variety. Hence, if a sheaf becomes reducible modulo a prime $p$ then its characteristic variety is reducible. Similarly, if a sheaf has irreducible characteristic variety then it remains irreducible modulo all primes.

In Section 2, we state the main result. The proof is given in Section 3. We include a rather lengthy discussion of characteristic cycles and their basic properties from various points of view, which we hope will help clarify the notion. The most natural context for characteristic cycles is the setting of real (analytic) manifolds and real constructible sheaves which we discuss briefly in Section 3.5. In Section 4, we discuss some examples.

In 2010 on the Isle of Skye the second author gave a talk about the first examples (in types $B_{2}$ and $A_{7}$ ) of decomposition numbers of intersection cohomology complexes of Schubert varieties. Michael Finkelberg remarked that these examples coincided with examples due to Tanisaki and Kashiwara-Saito of reducible characteristic varieties, and asked if there was a relation. We would like to thank him for his observation and question. We would also like to thank Tom Braden for useful correspondence and verifying that a certain Schubert variety in type $A_{7}$ has reducible characteristic variety, which led us to believe beyond reasonable doubt that there was something going on!

Received by the editors November 17, 2012. 


\section{Statement of the theorem}

Let $X$ denote a smooth complex algebraic variety equipped with a Whitney stratification

$$
X=\bigsqcup_{\lambda \in \Lambda} X_{\lambda}
$$

by locally closed connected smooth subvarieties. To each stratum $X_{\lambda} \subset X$ and local system $\mathcal{L}$ of free $\mathbb{Z}$-modules on $X_{\lambda}$ one may associate two objects:

(i) $\mathcal{I C}\left(\overline{X_{\lambda}}, E_{\mathcal{L}}\right)$ : the intersection cohomology $\mathcal{D}_{X}$-module, which is a regular holonomic $\mathcal{D}_{X}$-module extending $E_{\mathcal{L}}$, a vector bundle with flat connection on $X_{\lambda}$ whose monodromy is given by $\mathcal{L} \otimes_{\mathbb{Z}} \mathbb{C}$.

(ii) $\operatorname{IC}\left(\overline{X_{\lambda}}, \mathcal{L}\right)$ : the extension by zero to $X$ of the intersection cohomlogy complex extending the local system $\mathcal{L}$ on $X_{\lambda}$. This is an object in $\mathbf{P}_{\Lambda}(X, \mathbb{Z})$, the abelian category of $\Lambda$-constructible perverse sheaves on $X$ with coefficients in $\mathbb{Z}$.

Associated to the above two objects are two basic problems:

(i) On $\mathcal{I C}\left(\overline{X_{\lambda}}, E_{\mathcal{L}}\right)$ one may choose a good filtration $F$. The associated graded is then a sheaf of modules over grD $\mathcal{D}_{X}=\mathcal{O}_{T^{*} X}$ with support contained in the union of the conormal bundles to the strata. Taking multiplicities along each conormal bundle gives rise to the characteristic cycle

$$
\mathrm{CC}\left(\mathcal{I C}\left(\overline{X_{\lambda}}, E_{\mathcal{L}}\right)\right)=\sum_{\mu} m_{\lambda \mu}^{\mathcal{L}}\left[\overline{T_{X_{\mu}}^{*} X}\right]
$$

A basic problem in the theory of $\mathcal{D}$-modules is the calculation of the multiplicities $m_{\lambda \mu}^{\mathcal{L}}$.

(ii) Fix a prime number $p$ and consider $\mathbf{I C}\left(\overline{X_{\lambda}}, \mathcal{L}\right) \stackrel{L}{\otimes_{\mathbb{Z}}} \mathbb{F}_{p}$ the reduction modulo $p$ of $\mathbf{I C}\left(\overline{X_{\lambda}}, \mathcal{L}\right)$. It is a perverse sheaf with $\mathbb{F}_{p}$-coefficients on $X$, which in general will not be simple. Given any local system $\mathcal{M}$ of $\mathbb{F}_{p}$-vector spaces on $X_{\mu}$ the second basic problem asks for the decomposition number:

$$
d_{\lambda, \mathcal{L}, \mu, \mathcal{M}}^{p}=\left[\mathbf{I C}\left(\overline{X_{\lambda}}, \mathcal{L}\right) \stackrel{L}{\otimes}_{\mathbb{Z}} \mathbb{F}_{p}: \mathbf{I C}\left(\overline{X_{\mu}}, \mathcal{M}\right)\right] .
$$

This question is related to interesting questions in representation theory. For example, in both the geometric Satake equivalence [12] and the modular Springer correspondence [4] the above (topological) problem is equivalent to the (algebraic) problem of determining the decomposition numbers of standard modules.

Let rk $\mathcal{M}$ denote the rank of the local system $\mathcal{M}$. Then:

Theorem 2.1. $\operatorname{rk} \mathcal{M} \cdot d_{\lambda, \mathcal{L}, \mu, \mathcal{M}}^{p} \leq m_{\lambda \mu}^{\mathcal{L}}$.

Recall that the characteristic variety of $\mathcal{I C}\left(\overline{X_{\lambda}}, E_{\mathcal{L}}\right)$ is defined as the support of $\operatorname{CC}\left(\mathcal{I C}\left(\overline{X_{\lambda}}\right), E_{\mathcal{L}}\right)$. The above theorem has the following consequence:

Corollary 2.2. Suppose that the characteristic variety of $\mathcal{I C}\left(\overline{X_{\lambda}}, E_{\mathcal{L}}\right)$ is irreducible. Then $\mathbf{I C}\left(\overline{X_{\lambda}}, \mathcal{L}\right) \stackrel{L}{\otimes_{\mathbb{Z}}} \mathbb{F}_{p} \cong \mathbf{I C}\left(\overline{X_{\lambda}}, \mathcal{L} \stackrel{L}{\otimes_{\mathbb{Z}}} \mathbb{F}_{p}\right)$ for all primes $p$. Hence, $\mathbf{I C}\left(\overline{X_{\lambda}}, \mathcal{L}\right) \stackrel{L}{\otimes_{\mathbb{Z}}} \mathbb{F}_{p}$ is simple if $\mathcal{L} \stackrel{L}{\otimes}_{\mathbb{Z}} \mathbb{F}_{p}$ is. 


\section{Characteristic cycles and the proof}

3.1. The Euler characteristic: Let $\mathbb{k}$ denote either $\mathbb{Z}, \mathbb{C}$ or $\mathbb{F}_{p}$ and let $D_{c}^{b}(p t, \mathbb{k})$ denote the bounded derived category of constructible sheaves of $\mathbb{k}$-modules on a point, also known as the full subcategory of the bounded derived category of $\mathbb{k}$-modules consisting of complexes with finitely generated cohomology. Let $K_{\mathbb{k}}$ denote the Grothendieck group of $D_{c}^{b}(p t, \mathbb{k})$. We have a canonical isomorphism

$$
\chi: K_{\mathbb{k}} \stackrel{\sim}{\rightarrow} \mathbb{Z}
$$

fixed by declaring $\chi([\mathbb{k}])=1$, where $[\mathbb{k}]$ denote the class of the free module of rank 1 (placed in degree zero). Of course $\chi$ is just the Euler characteristic if $\mathbb{k}$ is a field, and is the alternating sum of the ranks if $\mathbb{k}=\mathbb{Z}$.

Consider the functors of extension of scalars:

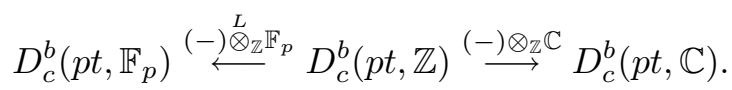

These functors are exact (in the triangulated sense) and hence induce maps on the corresponding Grothendieck groups:

$$
K_{\mathbb{F}_{p}} \stackrel{f}{\longleftarrow} K_{\mathbb{Z}} \stackrel{c}{\longrightarrow} K_{\mathbb{C}}
$$

These functors preserve the class of free module of rank 1. Hence:

Lemma 3.1. $f$ and $c$ are isomorphisms commuting with $\chi$.

3.2. The characteristic cycle of a constructible sheaf: Recall that $X=\bigsqcup X_{\lambda}$ is a smooth Whitney stratified complex algebraic variety. We let $T^{*} X$ denote the cotangent bundle of $X, \pi: T^{*} X \rightarrow X$ the canonical projection and $T_{\lambda}^{*} X:=T_{X_{\lambda}}^{*} X$ the conormal bundle to $X_{\lambda} \subset X$. A covector $\xi \in T_{\lambda}^{*} X$ is called non-degenerate if $\xi \notin \overline{T_{\mu}^{*} X}$ for all $\mu \neq \lambda$.

As above let $\mathbb{k} \in\left\{\mathbb{Z}, \mathbb{C}, \mathbb{F}_{p}\right\}$ and denote by $D_{\Lambda}^{b}(X ; \mathbb{k})$ the $\Lambda$-constructible derived category: this is the full subcategory of the bounded derived category of sheaves of $\mathbb{k}$-modules consisting of complexes whose cohomology sheaves are constructible with respect to the stratification $\Lambda$.

Now fix a constructible complex $\mathcal{F} \in D_{\Lambda}^{b}(X ; \mathbb{k})$. To $\mathcal{F}$ we wish to associate its characteristic cycle, which is a $\mathbb{Z}$-linear combination of the cycles $\left[\overline{T_{\lambda}^{*} X}\right]$ for all $\lambda \in \Lambda$. To this end fix a stratum $X_{\lambda}$, a point $x \in X_{\lambda}$ and a non-degenerate covector $\xi \in T_{\lambda}^{*} X$ such that $\pi(\xi)=x$. Then there exists a neighbourhood $U$ of $x$ and a holomorphic function $\phi: U \rightarrow \mathbb{C}$ such that

(i) $\phi(x)=0$ and $d \phi_{x}=\xi$,

(ii) The image of $d \phi$ intersects $\bigcup T_{\mu}^{*} X$ only at $\xi$, and this intersection is transverse.

Now set

$$
A_{\xi}(\mathcal{F}):=R \Gamma_{\{\operatorname{Re} \phi \geq 0\}}(\mathcal{F})_{x} \in D_{\Lambda}^{b}(\{x\} ; \mathbb{k})
$$

where $\{\operatorname{Re} \phi \geq 0\}:=\{x \in X \mid \operatorname{Re} \phi(x) \geq 0\}$. The complex $A_{\xi}(\mathcal{F})$ is independent of the choice of $\phi$ and its Euler characteristic

$$
m_{\lambda}(\mathcal{F}):=\chi\left(A_{\xi}(\mathcal{F})\right)
$$


depends only $\lambda$. One then defines the characteristic cycle as

$$
\mathrm{CC}(\mathcal{F}):=\sum_{\lambda \in \Lambda} m_{\lambda}(\mathcal{F})\left[\overline{T_{\lambda}^{*} X}\right] \in \bigoplus_{\lambda \in \Lambda} \mathbb{Z}\left[\overline{T_{\lambda}^{*} X}\right]
$$

For a more concrete description of $A_{\xi}(\mathcal{F})$; see for example [13, Section 2].

\subsection{Basic properties of the characteristic cycle:}

(i) The characteristic cycle factors through the Grothendieck group of $D_{\Lambda}^{b}(X ; \mathbb{k})$ : given a distinguished triangle

$$
\mathcal{F}^{\prime} \rightarrow \mathcal{F} \rightarrow \mathcal{F}^{\prime \prime} \stackrel{[1]}{\longrightarrow}
$$

then $\mathrm{CC}(\mathcal{F})=\mathrm{CC}\left(\mathcal{F}^{\prime}\right)+\mathrm{CC}\left(\mathcal{F}^{\prime \prime}\right)$. (This is immediate from the definition.)

(ii) Given $\mathcal{F} \in D_{\Lambda}^{b}(X, \mathbb{k})$ and $X_{\lambda}$ open in the support of $\mathcal{F}$ then for any non-degenerate $\xi \in T_{\lambda}^{*} X$ we have

$$
A_{\xi}(\mathcal{F}) \cong \mathcal{F}_{x}\left[-d_{\lambda}\right]
$$

where $d_{\lambda}$ denotes the complex dimension of $X_{\lambda}$. (This may be checked directly from the definition.) In particular, if $\mathcal{L}$ denotes a local system of free $\mathbb{k}$ modules on $X_{\lambda}$ and $\mathbf{I C}\left(\overline{X_{\lambda}} ; \mathcal{L}\right)$ denotes the intersection cohomology extension then $m_{\lambda}\left(\mathbf{I C}\left(\overline{X_{\lambda}} ; \mathcal{L}\right)\right)=\operatorname{rk} \mathcal{L}$, the rank of $\mathcal{L}$.

(iii) Let $\mathbf{P}_{\Lambda}(X ; \mathbb{k}) \subset D_{\Lambda}^{b}(X ; \mathbb{k})$ denote the abelian subcategory of perverse sheaves (see [1]). Then $\mathcal{F} \in D_{\Lambda}^{b}(X ; \mathbb{k})$ is perverse if and only if for all $\lambda \in \Lambda$ and non-degenerate $\xi \in T_{\lambda}^{*} X$ the complex $A_{\xi}(\mathcal{F})$ is concentrated in degree 0 , see [7, Theorem 10.3.12]. Indeed, one can argue as in [3, Theorem 6.4] to show that $A_{\xi}(\mathcal{F})$ is concentrated in degree 0 if $\mathcal{F}$ is perverse. The other direction follows from the fact that if $A_{\xi}(\mathcal{F})=0$ for all non-degenerate $\xi \in T_{\lambda}^{*} X$ then $\mathcal{F}=0$. In particular, for $\mathcal{F} \in \mathbf{P}_{\Lambda}(X ; \mathbb{k})$ we have

$$
\mathrm{CC}(\mathcal{F}) \in \bigoplus_{\lambda} \mathbb{Z}_{\geq 0}\left[\overline{T_{\lambda}^{*} X}\right]
$$

(iv) The characteristic cycle operation commutes with the Riemann-Hilbert correspondence. Let $\mathcal{D}_{X}-\operatorname{Mod}_{\Lambda}$ denote the abelian category of regular holonomic $\mathcal{D}_{X}$-modules whose characteristic variety is contained in $\bigcup \overline{T_{\lambda}^{*} X}$. Recall that the Riemman-Hilbert correspondence gives an equivalence of abelian categories

$$
R H: \mathcal{D}_{X}-\operatorname{Mod}_{\Lambda} \stackrel{\sim}{\rightarrow} \mathbf{P}_{\Lambda}(X ; \mathbb{C}) .
$$

Given any $\mathcal{D}_{X}$-module $\mathcal{M}$ one can define its characteristic cycle using the theory of good filtrations. We have

$$
\mathrm{CC}(\mathcal{M})=\mathrm{CC}(R H(\mathcal{M})) .
$$

This is a theorem due to Kashiwara [5, Section 8.2]. See the final pages of [14] for a short proof of this fact. The basic idea is that using results of Ginzburg [2] and [13] both sides can be seen to agree if $\mathcal{M}$ is the $\mathcal{D}$-module direct image of a vector bundle with flat connection on a locally closed subvariety, and such direct images generate the Grothendieck group of all holonomic $\mathcal{D}$-modules. 
(v) The characteristic cycle commutes with extension of scalars: If $\mathbb{k} \in\left\{\mathbb{C}, \mathbb{F}_{p}\right\}$ and $\mathcal{F} \in D_{\Lambda}^{b}(X ; \mathbb{Z})$ then

$$
\mathrm{CC}(\mathcal{F})=\mathrm{CC}\left(\mathcal{F} \otimes_{\mathbb{Z}}^{L} \mathbb{k}\right) .
$$

This is immediate from Lemma 3.1 and the fact that the functors $A_{\xi}(-)$ and $(-) \otimes_{\mathbb{Z}}^{L} \mathbb{k}$ commute up to natural isomorphism.

3.4. Proof of the theorem: Fix $\lambda \in \Lambda$ and a local system $\mathcal{L}$ of free $\mathbb{Z}$-modules on $X_{\lambda}$. Let $\mathbf{I C}\left(\overline{X_{\lambda}}, \mathcal{L}\right)$ denote the intersection cohomology complex of $\overline{X_{\lambda}}$ with coefficients in $\mathcal{L}$. We have

$$
\mathbf{I C}\left(\overline{X_{\lambda}}, \mathcal{L}\right) \otimes_{\mathbb{Z}} \mathbb{C} \cong \mathbf{I C}\left(\overline{X_{\lambda}}, \mathcal{L} \otimes_{\mathbb{Z}} \mathbb{C}\right)
$$

Fix a prime $p$. In the Grothendieck group of $\mathbf{P}_{\Lambda}\left(X, \mathbb{F}_{p}\right)$ we can write

$$
\left[\mathbf{I C}\left(\overline{X_{\lambda}}, \mathcal{L}\right) \otimes_{\mathbb{Z}}^{L} \mathbb{F}_{p}\right]=\sum d_{\lambda, \mathcal{L}, \mu, \mathcal{M}}^{p}\left[\mathbf{I C}\left(\overline{X_{\mu}} ; \mathcal{M}\right)\right]
$$

where the sum runs over all pairs $\mu, \mathcal{M}$ where $\mu \in \Lambda$ and $\mathcal{M}$ is a irreducible local system of $\mathbb{F}_{p}$-vector spaces on $X_{\mu}$. Applying CC and using (i), (ii) and (iii) above we are led to the inequalities

$$
m_{\mu}\left(\mathbf{I C}\left(\overline{X_{\lambda}}, \mathcal{L}\right) \otimes_{\mathbb{Z}}^{L} \mathbb{F}_{p}\right) \geq \operatorname{rk} \mathcal{M} \cdot d_{\lambda, \mathcal{L}, \mu, \mathcal{M}}^{p} .
$$

By (iv) and (v) we get (with notation as in Section 2)

$$
\begin{aligned}
\operatorname{CC}\left(\mathcal{I C}\left(\overline{X_{\lambda}}, E_{\mathcal{L}}\right)\right)=\operatorname{CC}\left(\mathbf{I C}\left(\overline{X_{\lambda}} ; \mathcal{L} \otimes_{\mathbb{Z}} \mathbb{C}\right)=\right. \\
\left.\operatorname{CC}\left(\mathbf{I C}\left(\overline{X_{\lambda}}, \mathcal{L}\right) \otimes \mathbb{C}\right)\right)=\operatorname{CC}\left(\mathbf{I C}\left(\overline{X_{\lambda}} ; \mathcal{L}\right) \otimes_{\mathbb{Z}}^{L} \mathbb{F}_{p}\right) .
\end{aligned}
$$

Hence

$$
m_{\lambda \mu}^{\mathcal{L}}=m_{\mu}\left(\mathbf{I C}\left(\overline{X_{\lambda}}, \mathcal{L}\right) \otimes_{\mathbb{Z}}^{L} \mathbb{F}_{p}\right)
$$

which completes the proof.

3.5. Remarks on the real analytic case. Let $X$ to be a real analytic manifold and consider $D_{c}^{b}(X ; \mathbb{k})$ the bounded derived category of sub-analytically constructible sheaves. In this case the characteristic cycle has been defined by Kashiwara [5, Section 3]. It coincides with the previous definition in the complex case.

In the real case we get an isomorphism

$$
\mathrm{CC}: K(X ; \mathbb{k}) \stackrel{\sim}{\rightarrow} \mathcal{L}_{X}
$$

where $K(X ; \mathbb{k})$ denotes the Grothendieck group of $D_{c}^{b}(X ; \mathbb{k})$ and $\mathcal{L}_{X}$ denotes the group of $\mathbb{R}^{+}$-invariant, sub-analytic Lagrangian cycles on $T^{*} X$ with integral coefficients. (One way of seeing this is to use the fact that any stratification can be refined to a triangulation, where monodromy no longer plays a role.)

Remark 3.1. Using Kashiwara's definition it is not immediately obvious that one obtains a cycle. However, for standard sheaves on simplices this follows by the limit construction given in [13]. As these objects generate $K(X ; \mathbb{k})$ it follows that one obtains a cycle in general. 
Consider the functors of extension of scalars:

$$
D_{c}^{b}\left(X, \mathbb{F}_{p}\right) \stackrel{(-) \stackrel{L}{\otimes_{\mathbb{Z}}} \mathbb{F}_{p}}{\longleftarrow} D_{c}^{b}(X, \mathbb{Z}) \stackrel{(-) \otimes_{\mathbb{Z}} \mathbb{C}}{\longrightarrow} D_{c}^{b}(X, \mathbb{C}) .
$$

They induce maps on the corresponding Grothendieck groups

$$
K\left(X ; \mathbb{F}_{p}\right) \stackrel{f}{\leftarrow} K(X ; \mathbb{Z}) \stackrel{c}{\rightarrow} K(X ; \mathbb{C})
$$

which commute with CC. We summarize this discussion by an analogue of Lemma 3.1:

Lemma 3.2. $f$ and $c$ are isomorphisms commuting with $\mathrm{CC}$ and hence all the groups $K\left(X ; \mathbb{F}_{p}\right), K(X ; \mathbb{Z}), K(X ; \mathbb{C})$, and $\mathcal{L}_{X}$ are canonically isomorphic.

(One can see directly that $f$ and $c$ are isomorphisms by again refining to a triangulation.)

Finally, let us note that an alternative definition of the characteristic cycle has been given by Kashiwara and Schapira [6, Section 9.4] under the assumption that $\mathbb{k}$ is a field of characteristic zero. It has the disadvantage that it does not work well with finite characteristic coefficients.

\section{Examples}

4.1. The flag variety of type $\boldsymbol{B}_{2}$ : Let $G$ be a simple complex algebraic group of type $B_{2}, B \subset G$ a Borel subgroup and $X=G / B$ its flag variety. Let $W$ be the Weyl group of $G$ with simple reflections $s$ and $t$, corresponding to the short and long simple roots respectively. Consider the Bruhat decompositon

$$
X=\bigoplus_{w \in W} B w B / B=\bigsqcup_{w \in W} X_{w} .
$$

Then the Schubert varieties $\overline{X_{w}}$ are smooth unless $w=$ sts. In this case

$$
\mathrm{CC}\left(\mathcal{I C}\left(\overline{X_{s t s}}\right)\right)=\left[\overline{T_{s t s}^{*} X}\right]+\left[\overline{T_{s}^{*} X}\right]
$$

This is the first example of a reducible characteristic variety of a Schubert variety, and is due to Kashiwara and Tanisaki [8].

The singularity of $X_{\text {sts }}$ along $X_{s}$ is smoothly equivalent to a Kleinian surface singularity of type $A_{1}$. Hence, one has (see [4, Section 2.5.3])

$$
d_{s t s, s}^{p}= \begin{cases}1 & \text { if } p=2, \\ 0 & \text { otherwise }\end{cases}
$$

4.2. Flag varieties of type $\boldsymbol{A}$ : Let $G=S L_{n}(\mathbb{C}), B \subset G$ a Borel subgroup and $W=S_{n}$ be the Weyl group. As in the previous example we consider the Bruhat decomposition

$$
X=\bigoplus_{w \in W} B w B / B=\bigsqcup_{w \in W} X_{w} .
$$

Using the computer calculations in [17, Section 5.1] one can show there are no decomposition numbers if $n<8$. If $n=8$ then one can use [17, Section 5.2] to conclude that all decomposition numbers are zero except for 38 possible exceptions. Building of work of Braden [17, Appendix A] the second author has performed computer calculations to verify that $\mathbf{I C}\left(\overline{X_{w}} ; \mathbb{Z}\right) \otimes_{\mathbb{Z}}^{L} \mathbb{F}_{2}$ is not simple in these remaining 38 cases. Our main 
theorem now implies that all of these Schubert varieties have reducible characteristic variety.

One of these 38 Schubert varieties (the Schubert variety corresponding to the permutation 62845173) has a singularity smoothly equivalent to a singularity in a quiver variety of type $A_{5}$. This singularity was used by Kashiwara and Saito [6, Theorem 7.2.1] to provide a counterexample to a conjecture of Kazhdan and Lusztig on the irreducibility of characteristic varieties in type $A$ quiver varieties and Schubert varieties. The second author [16] has recently noticed that this example also gives a counterexample to the Kleshchev-Ram conjecture [10].

\section{Acknowledgments}

K. Vilonen was supported by NSF and by DARPA via AFOSR grant FA9550-08-10315. Both authors were supported by the EPSRC and would like to thank the MPI, Bonn for a productive research environemnt.

\section{References}

[1] A.A. Beyllinson, J. Bernstein and P. Deligne, Faisceaux pervers, in 'Analysis and topology on singular spaces, I' (Luminy, 1981), Vol. 100 of Astérisque, 5-171, Soc. Math. France, Paris (1982).

[2] V. Ginsburg, Characteristic varieties and vanishing cycles, Invent. Math. 84(2) (1986), 327-402.

[3] M. Goresky and R. MacPherson, Stratified Morse theory, Vol. 14 of Ergebnisse der Mathematik und ihrer Grenzgebiete (3) [Results in Mathematics and Related Areas (3)], Springer-Verlag, Berlin (1988), ISBN 3-540-17300-5.

[4] D. Juteau, Modular Springer correspondence and decomposition matrices, Ph.D. thesis, Université de Paris 7 (2007).

[5] M. Kashiwara, Index theorem for constructible sheaves, Astérisque (1985), no. 130, $193-209$. Differential systems and singularities (Luminy, 1983).

[6] M. Kashiwara and Y. Saito, Geometric construction of crystal bases, Duke Math. J. 89(1) (1997), 9-36.

[7] M. Kashiwara and P. Schapira, Sheaves on manifolds, Vol. 292 of Grundlehren der Mathematischen Wissenschaften [Fundamental Principles of Mathematical Sciences], Springer-Verlag, Berlin (1994), ISBN 3-540-51861-4. With a chapter in French by Christian Houzel, Corrected reprint of the 1990 original.

[8] M. Kashiwara and T. Tanisaki, The characteristic cycles of holonomic systems on a flag manifold related to the Weyl group algebra, Invent. Math. 77(1) (1984), 185-198.

[9] D. Kazhdan and G. Lusztig, A topological approach to Springer's representations, Adv. Math. 38(2) (1980), 222-228.

[10] A. Kleshchev and A. Ram, Representations of Khovanov-Lauda-Rouquier algebras and combinatorics of Lyndon words, Math. Ann. 349(4) (2011), 943-975.

[11] G. Lusztig, Quivers, perverse sheaves, and quantized enveloping algebras, J. Amer. Math. Soc. 4(2) (1991), 365-421.

[12] I. Mirković and K. Vilonen, Geometric Langlands duality and representations of algebraic groups over commutative rings, Ann. Math. (2) 166(1) (2007), 95-143.

[13] W. Schmid and K. Vilonen, Characteristic cycles of constructible sheaves, Invent. Math. 124(1-3) (1996), 451-502.

[14] - Characteristic cycles and wave front cycles of representations of reductive Lie groups, Ann. Math. (2) 151(3) (2000), 1071-1118.

[15] T. Tanisaki, Characteristic varieties of highest weight modules and primitive quotients, in Representations of Lie groups, Kyoto, Hiroshima, 1986, Vol. 14 of Adv. Stud. Pure Math., 1-30, Academic Press, Boston, MA (1988). 
[16] G. Williamson, On an analogue of the James conjecture, Preprint. 12 p.

[17] G. Williamson and T. Braden, Modular intersection cohomology complexes on flag varieties, Math. Z. 272(3-4) (2012), 697-727.

Department of Mathematics, Northwestern University, Evanston, IL 60208, USA, And Department of Mathematics, Helsinki University, Helsinki, Finland

E-mail address: vilonen@math.northwestern.edu, kari.vilonen@helsinki.fi

Max-Planck-Institut für Mathematik, Vivatsgasse 7, 53111 Bonn, Germany

E-mail address: geordie@mpim-bonn.mpg.de 